\title{
IMPACT OF COVID 19 ON PUBLIC SERVICE SUPPLY (IN THE CONTEXT OF LOCAL SELF-GOVERNMENT)
}

\author{
NANA RUSADZE \\ Doctor of Public Administration, Associate Professor \\ Akaki Tsereteli State University, Georgia \\ nana.rusadze@atsu.edu.ge
}

\section{EKATERINA ZAKARADZE}

Doctor of Public Administration, Invited Lecturer

Grigol Robakidze University, Georgia

ekaterine.zakaradze02@gruni.edu.ge

\author{
AZA IPSHIRADZE \\ Doctor of Economics, Professor \\ Kutaisi University, Georgia \\ aza.iphshiradze@unik.edu.ge
}

Abstract. The last two years in the history of mankind can be characterized as the beginning of a nation of difficulties caused by the spread of the coronavirus pandemic. The pandemic has broken all borders (political, legal, state) and overcoming the transboundary global crisis has necessitated the participation of all levels of government and the private sector.

The pandemic, along with human health, attacks the socio-economic sphere and poses obvious threats of economic depression, rising unemployment and poverty, limited social contacts, and many other negative events. This threat affects the state and municipal management and, consequently, the public services provided by them. Unfortunately, unlike trade and education, these types of services are largely impossible to digitize.

In the current situation, the attention of citizens and business representatives is drawn to the actions of the state, as its activities and management skills have acquired much more function. Meeting the needs of citizens in a pandemic requires the use of approaches based on new technologies, new forms of interaction and innovative methods of work.

The economic sustainability of public administration occupies an important place in public policy as a determinant of public sector modernization, support, optimization of functioning, and improvement of public services. This acquired a special load during the crisis caused by the coronapandemic.

The ongoing coronavirus pandemic dictates that it is necessary to develop new approaches to state intervention and public service delivery systems in all areas of public life in times of crisis in order to respond optimally to the needs of citizens.

KEYWORDS: CORONA VIRUS, PUBLIC SERVICES, OWN AUTHORITY, CRISIS, MUNICIPALITY.

For citation: Rusadze, N., Zakaradze, E., \& Ipshiradze, A. (2020). Impact of Covid 19 on Public Service Supply (In the Context of Local Self-Government). Globalization and Business. 12, 88-93. (In Georgian). https://doi.org/10.35945/gb.2021.12.012 


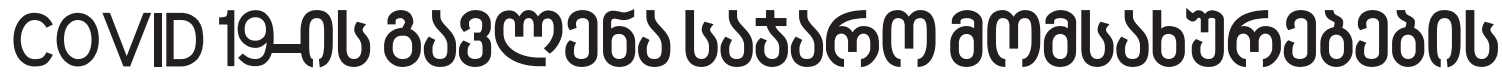

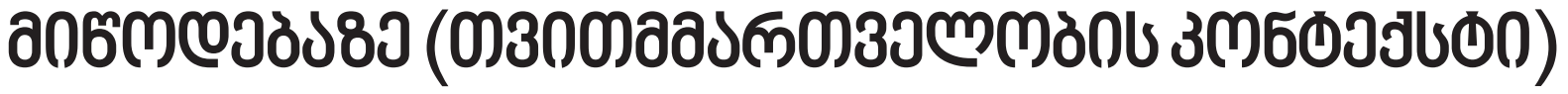

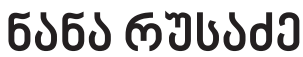

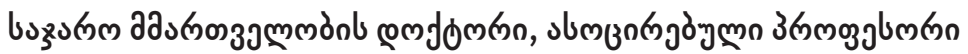

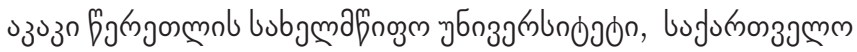

nana.rusadze@atsu.edu.ge

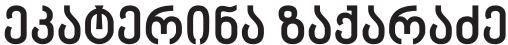

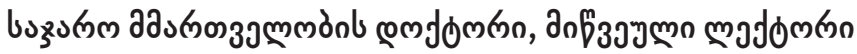

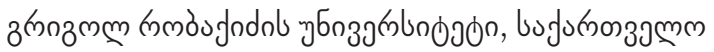

ekaterine.zakaradze02@gruni.edu.ge

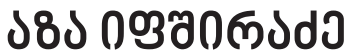

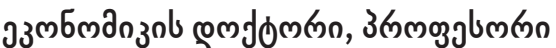

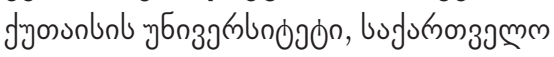 \\ aza.iphshiradze@unik.edu.ge
}

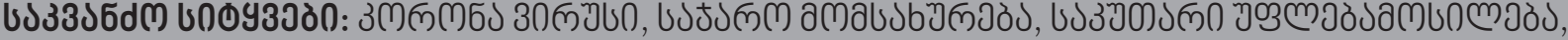

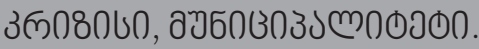

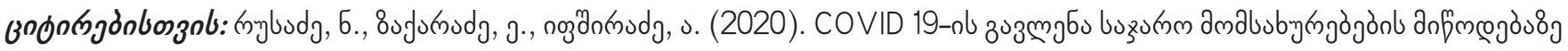

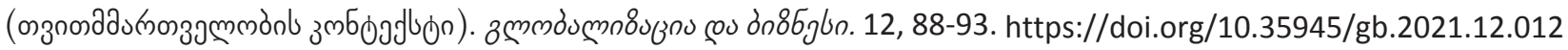

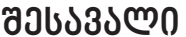

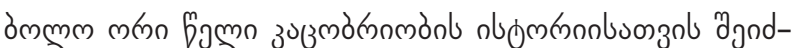

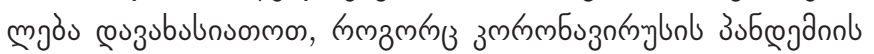

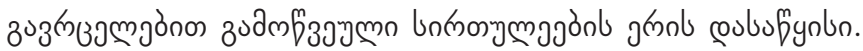

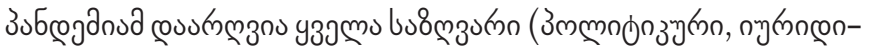

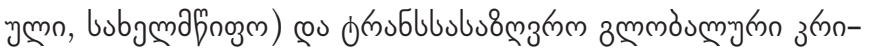

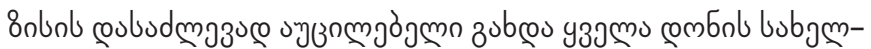

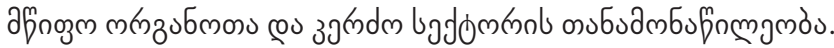

उง

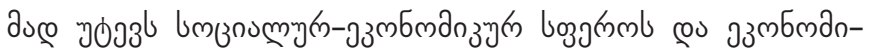

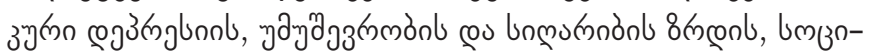

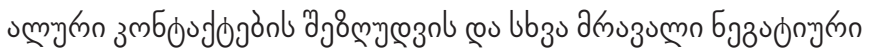

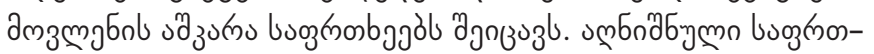

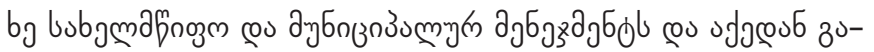

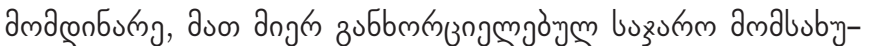

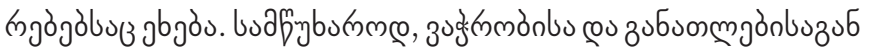

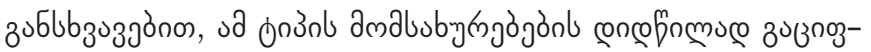

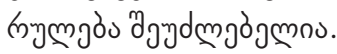

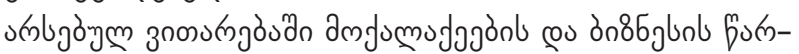

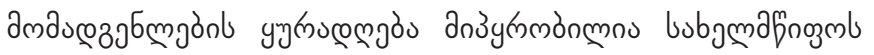

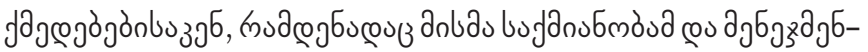

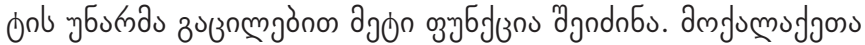

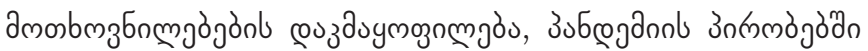

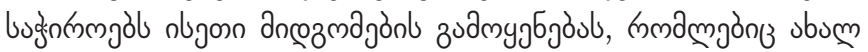

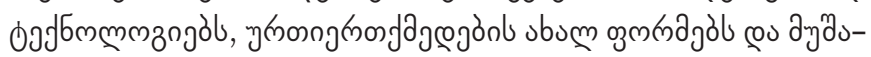

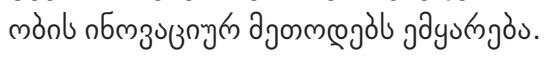

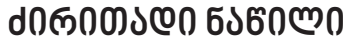

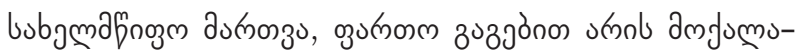

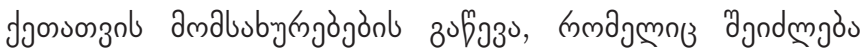

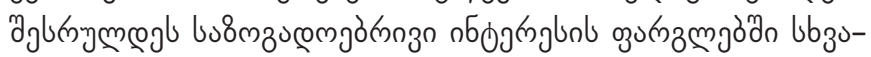

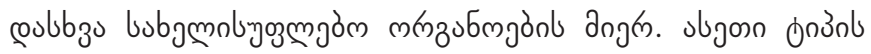

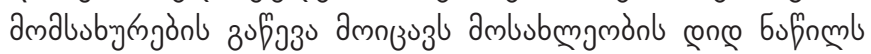

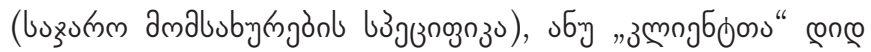
mumcjomós.

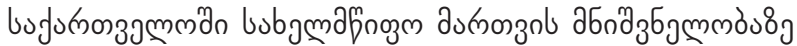

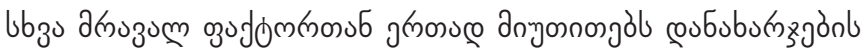

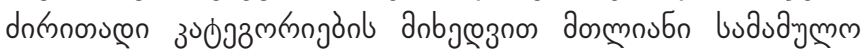

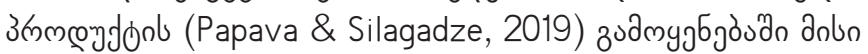

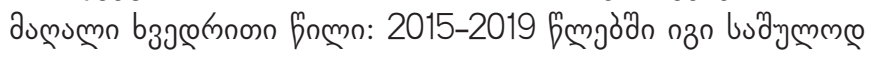

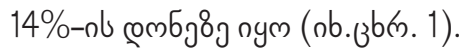




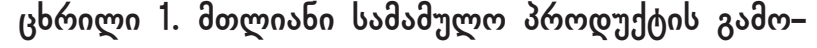
g9б

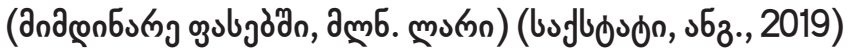

\begin{tabular}{|c|c|c|c|c|c|}
\hline 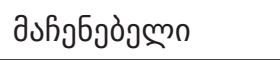 & 2015 & 2016 & 2017 & 2018 & 2019 \\
\hline 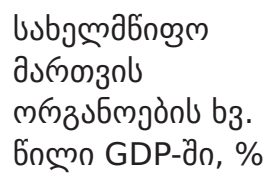 & 14,3 & 15,3 & 13,9 & 13,2 & 13,1 \\
\hline 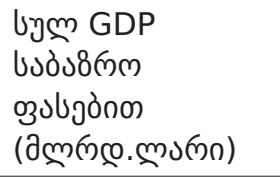 & 33,9 & 35,8 & 40,8 & 44,6 & 49,2 \\
\hline
\end{tabular}

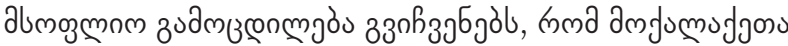

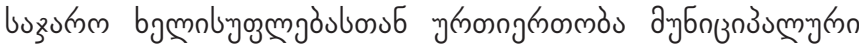

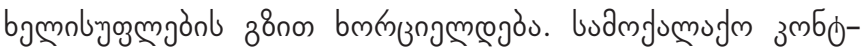

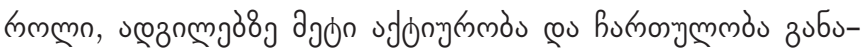

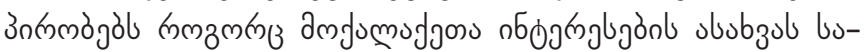

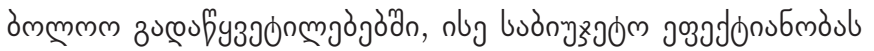
(Shergelashvili, 2017).

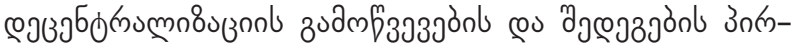

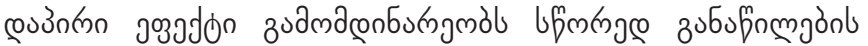

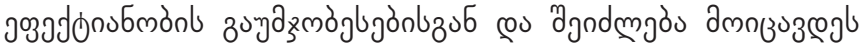

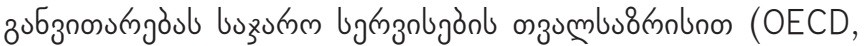
2019).

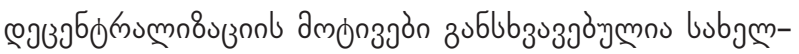

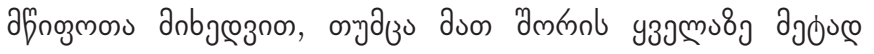

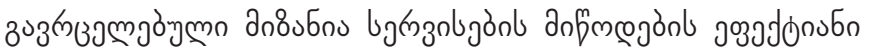

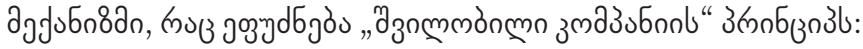

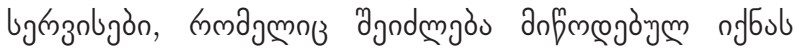

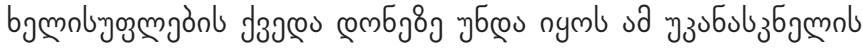

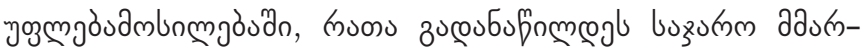

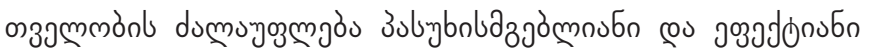

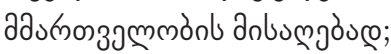

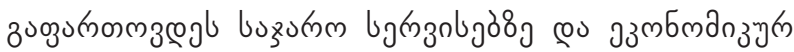

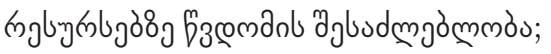

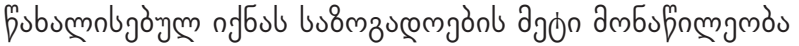

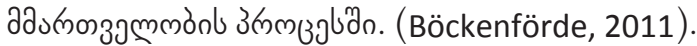

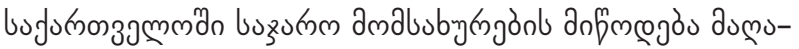

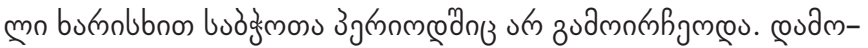

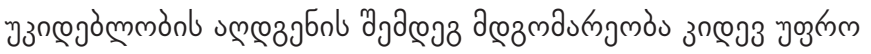

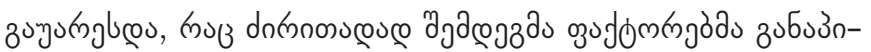
mmògl:

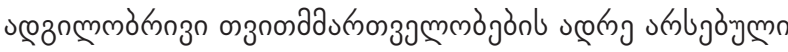

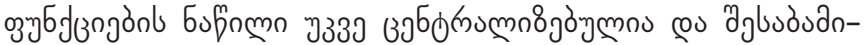

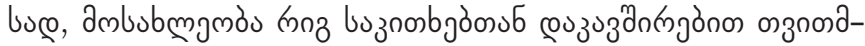

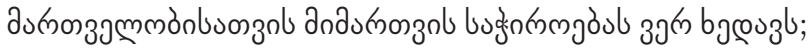

bu8mzuल

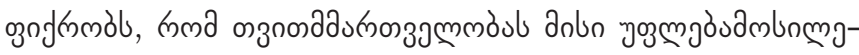

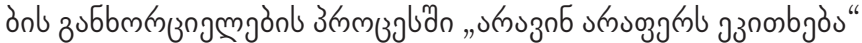

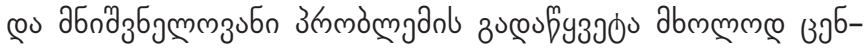

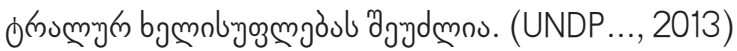

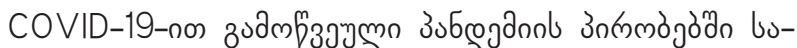

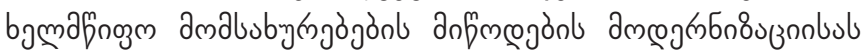

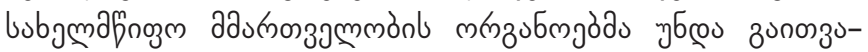

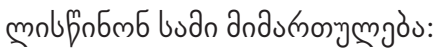

ง) dm fums

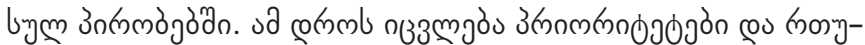

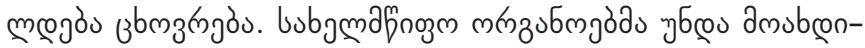

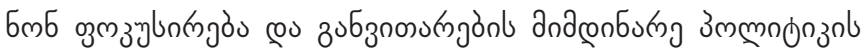

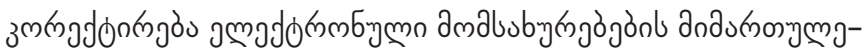

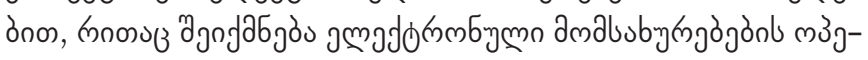

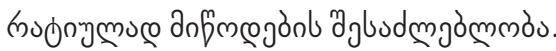

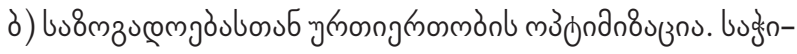

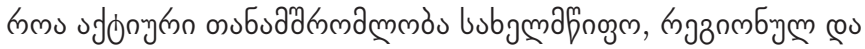

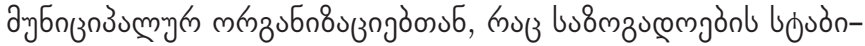

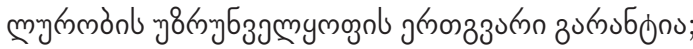

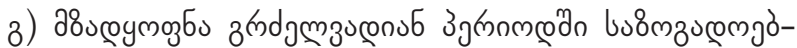

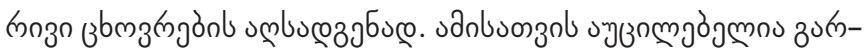

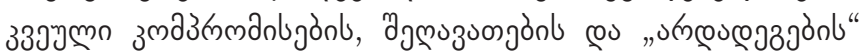
coffjbgos (UN/DESA Policy, 2020).

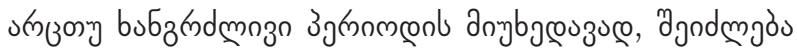

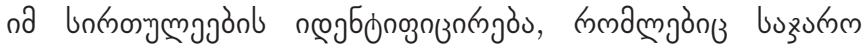

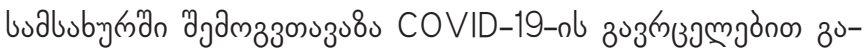

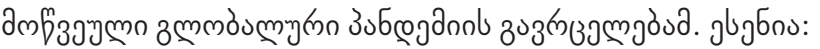

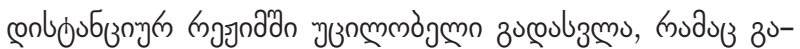

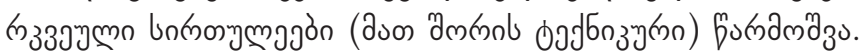

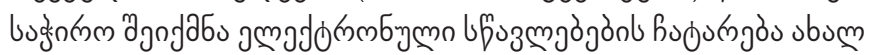

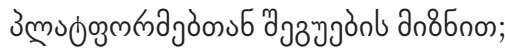

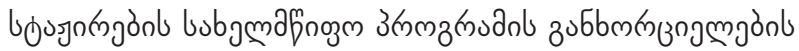

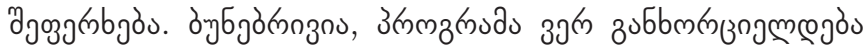

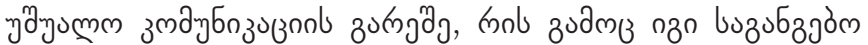

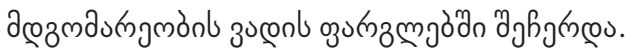

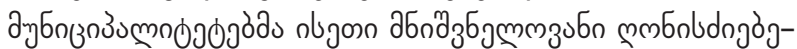

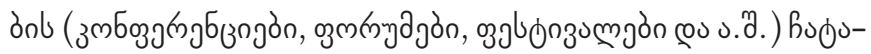

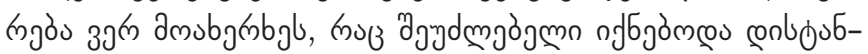

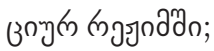

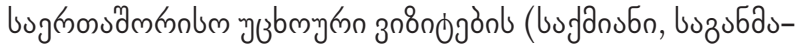

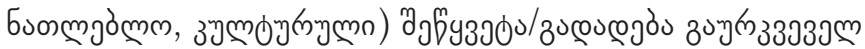

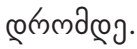

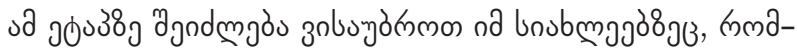

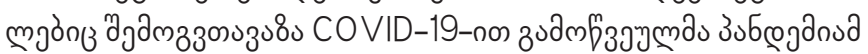
buzuknm buabubyng jadn:

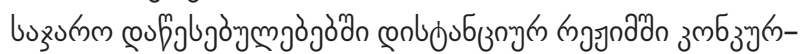
unl joüjànl Bopukngos;

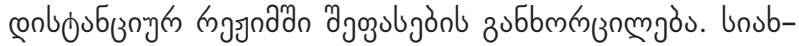

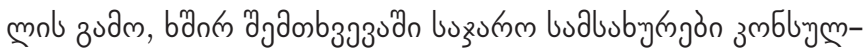
ouznjal n п

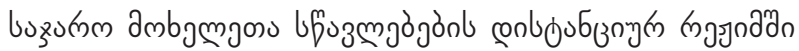




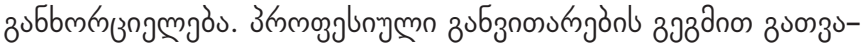

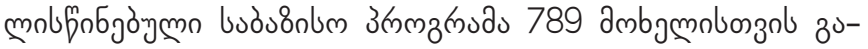

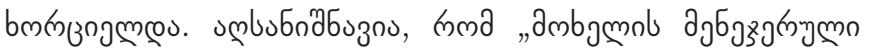

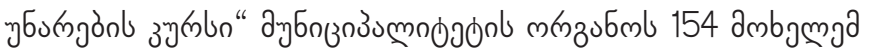

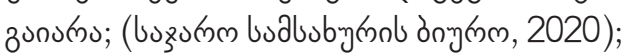

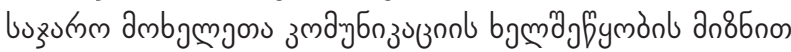

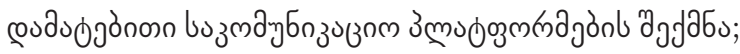

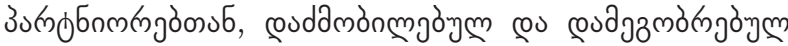

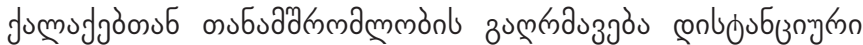
follon.

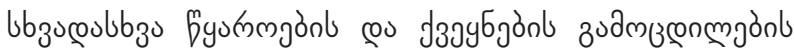

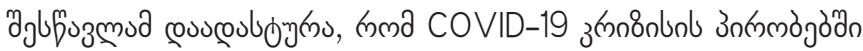

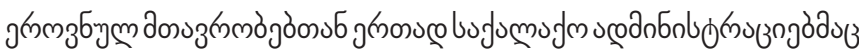

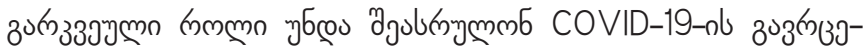

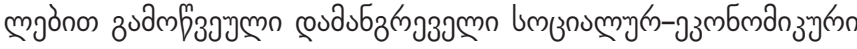

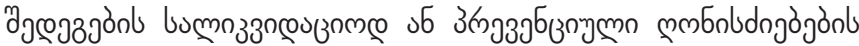

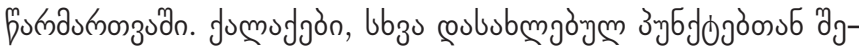

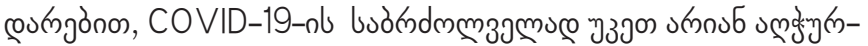

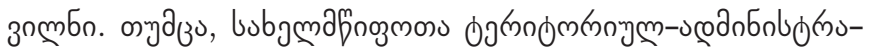

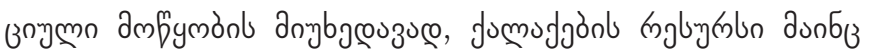

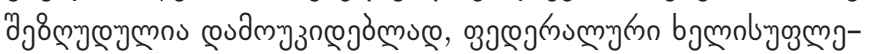

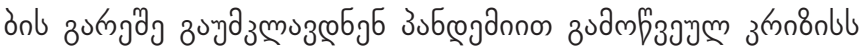
(Chikhladze et al., 2021).

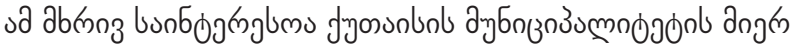

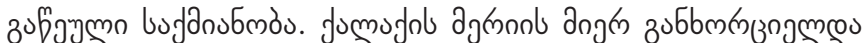

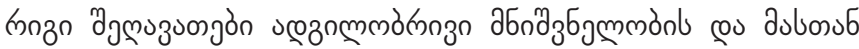
एuз з

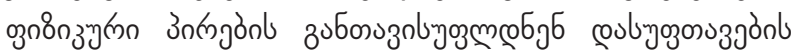

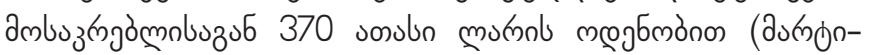

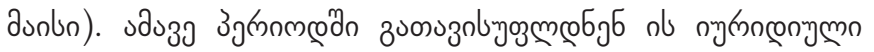

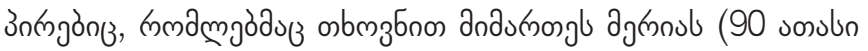
motron);

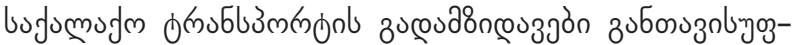

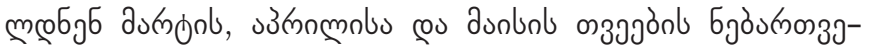

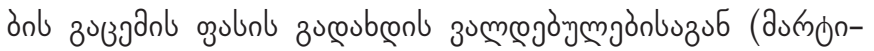

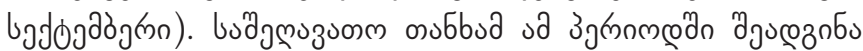
475,2 sosuln mutron. (Civil Service Bureau Activity Report, 2020);

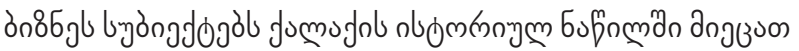

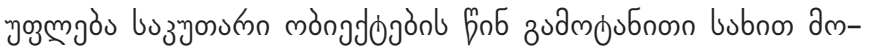

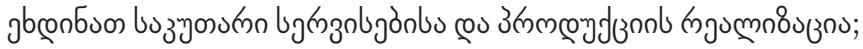

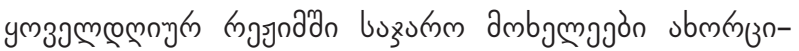

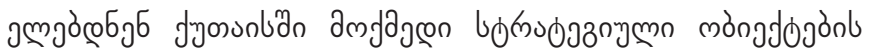

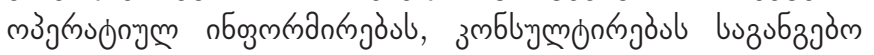

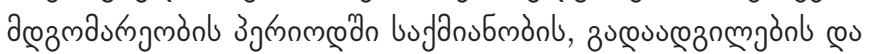

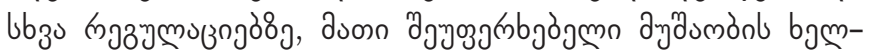

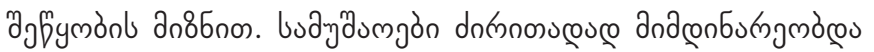

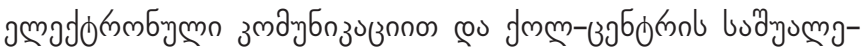

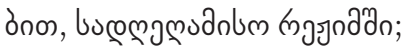

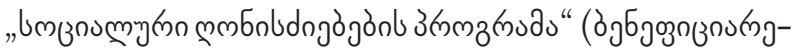

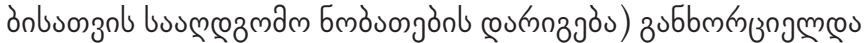

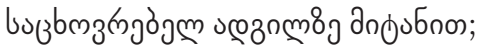

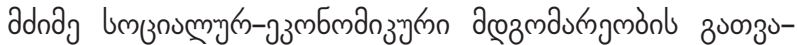

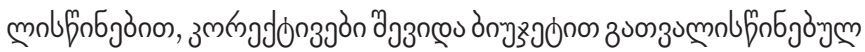

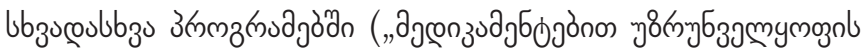

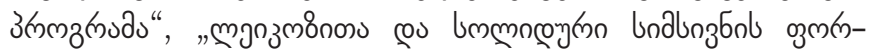

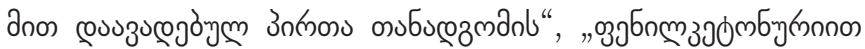

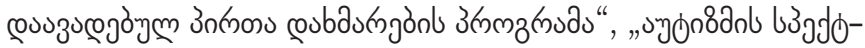

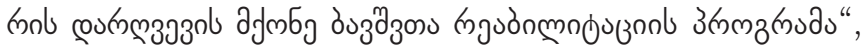

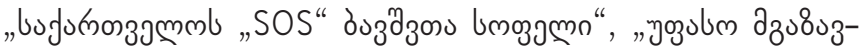

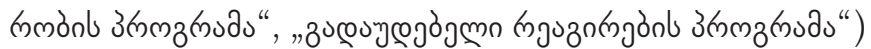

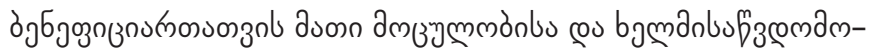
onl $308 \mathrm{~m}$ monl an 8 85nom;

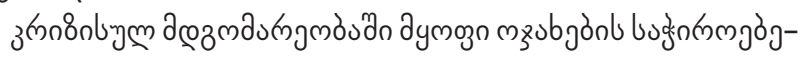

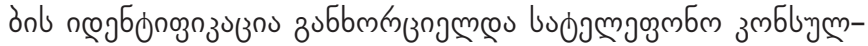

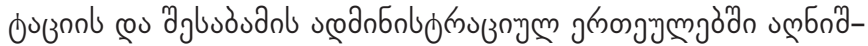

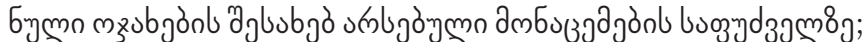

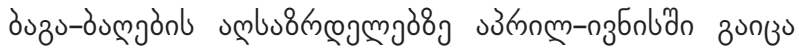

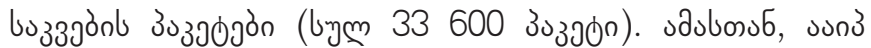

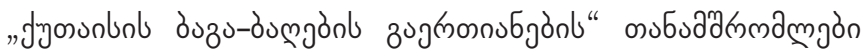

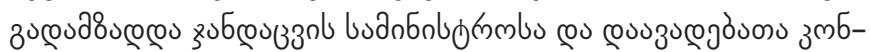

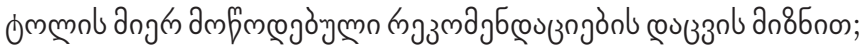

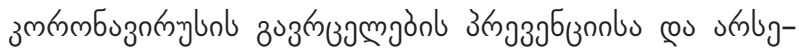

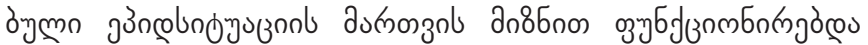

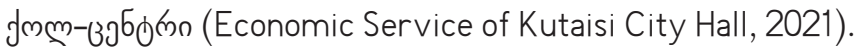

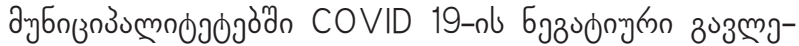

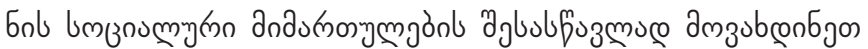

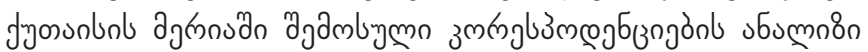
(nb. उbor. 2).

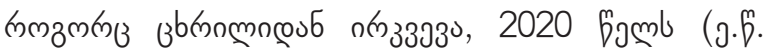

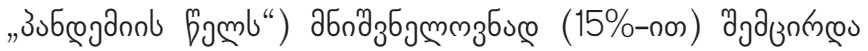

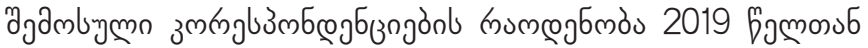

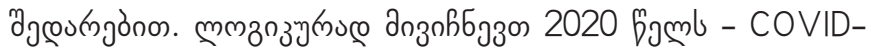

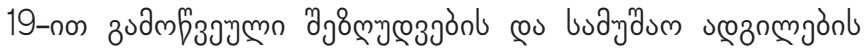

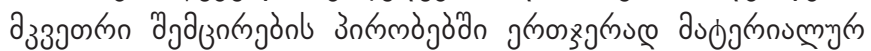

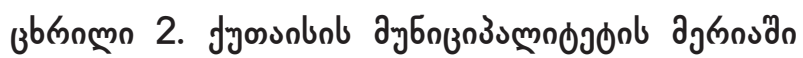

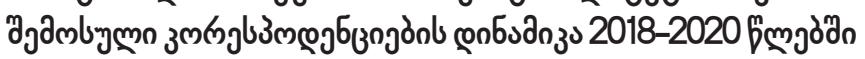

\begin{tabular}{|c|c|c|c|}
\hline \multirow[t]{2}{*}{ 6эmono } & \multirow{2}{*}{ 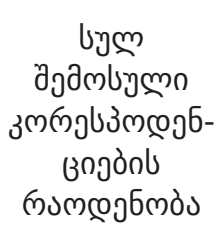 } & \multicolumn{2}{|c|}{ 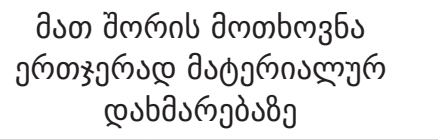 } \\
\hline & & byg & 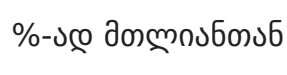 \\
\hline 2018 & 30109 & 1759 & 5,84 \\
\hline 2019 & 31868 & 1185 & 3,72 \\
\hline 2020 & 27193 & 2040 & 7,50 \\
\hline
\end{tabular}

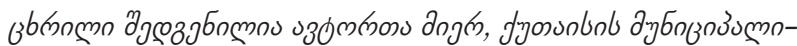

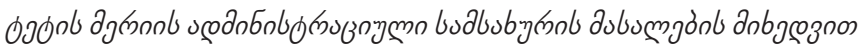




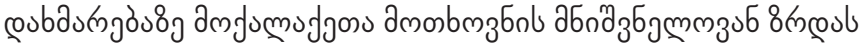

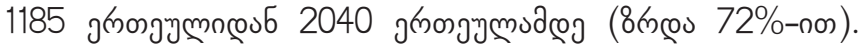

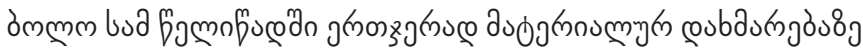

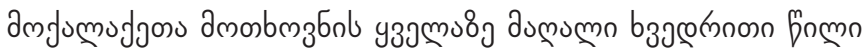

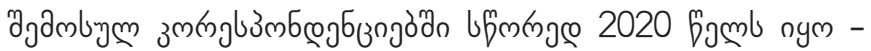
$7,5 \%$.

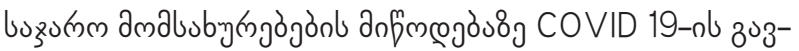

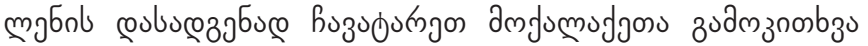

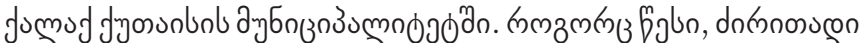

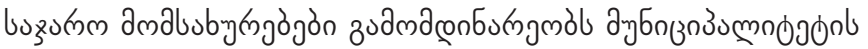

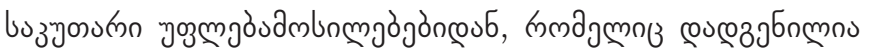

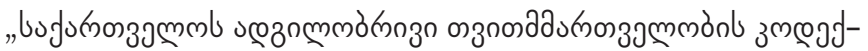
ঢno". Local (Self-Government Code, Article 16).

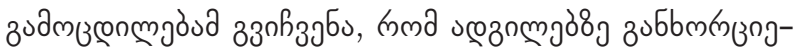

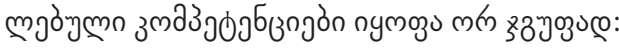

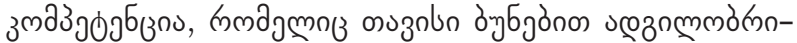

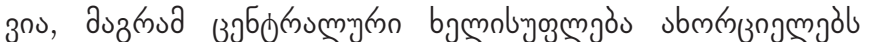

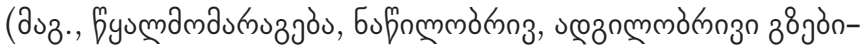

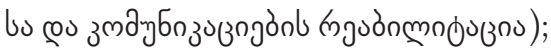

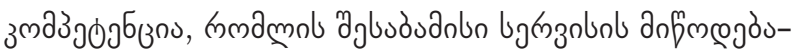

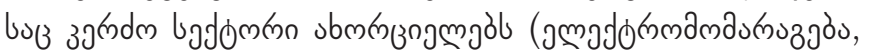

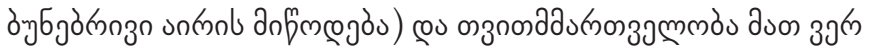

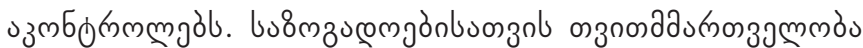

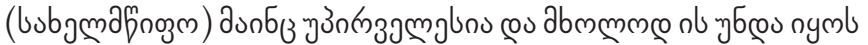

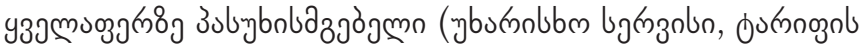

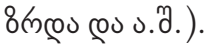

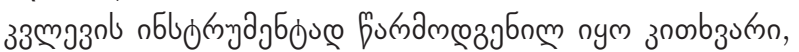

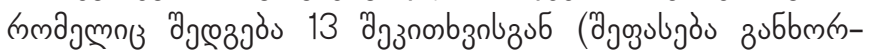

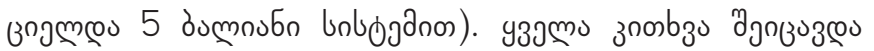

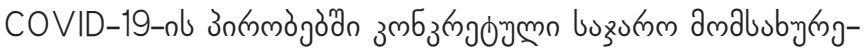

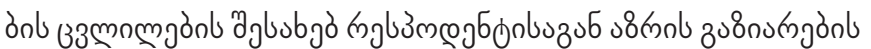

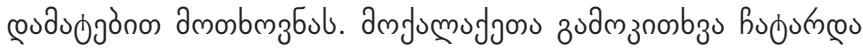

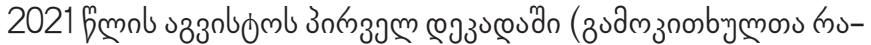

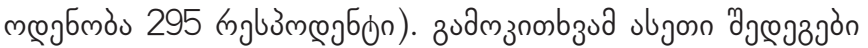
zudmuzmobu (nb. зbm. 3)

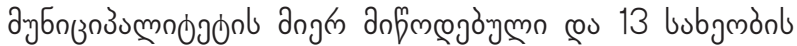

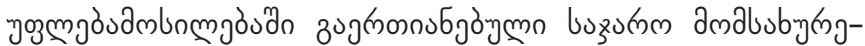

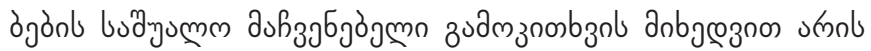

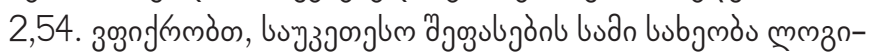

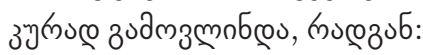

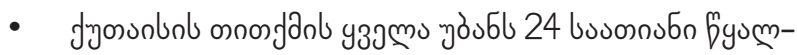

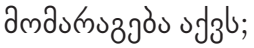

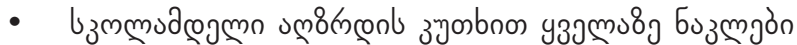

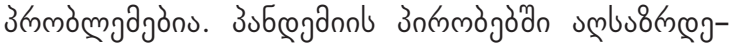

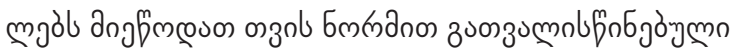

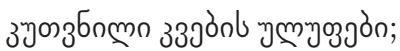

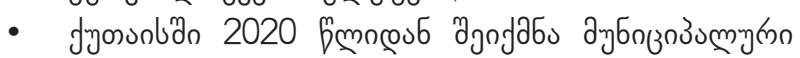
ónublismtron.

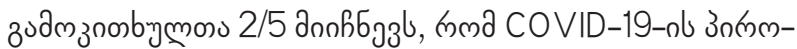

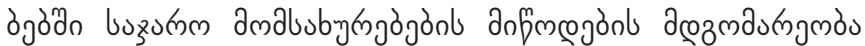

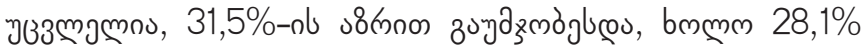

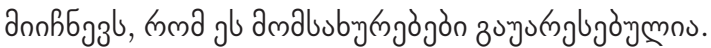

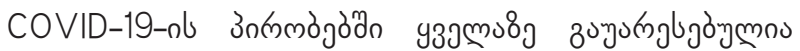
àn

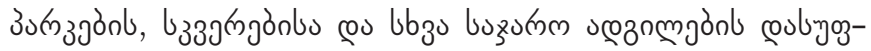
muзjos, zud

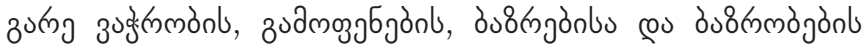

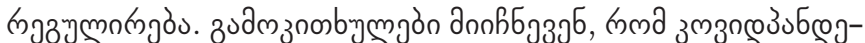

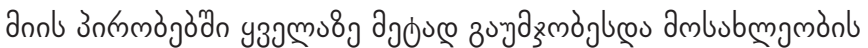

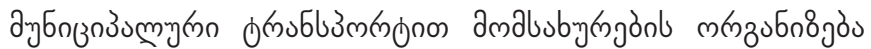

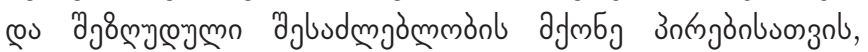

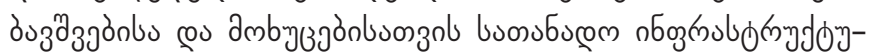
mols zubzonotrogas.

\section{๒ง৬3360д0}

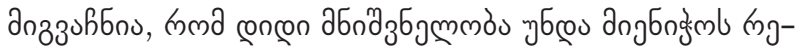

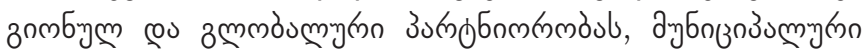

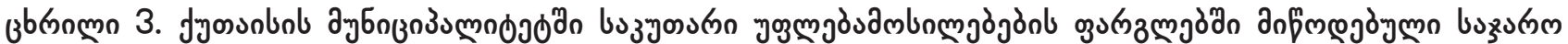
amabsbyg

\begin{tabular}{|c|c|c|c|c|c|}
\hline № & 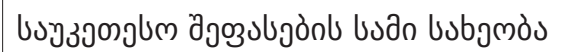 & 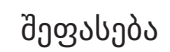 & № & 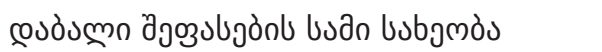 & 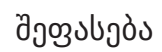 \\
\hline 1 & 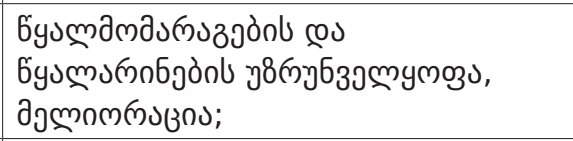 & 3,1 & I & 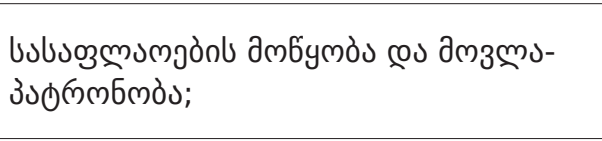 & 2,3 \\
\hline II & 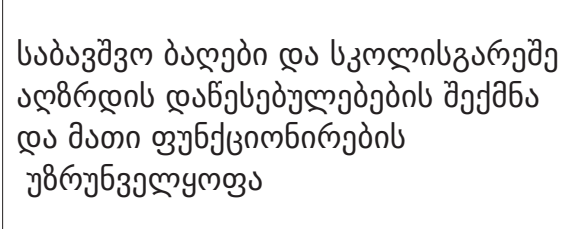 & 3,0 & $\|$ & 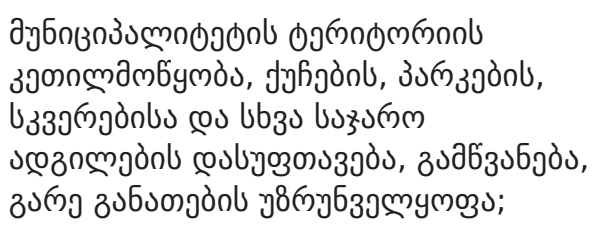 & 2,4 \\
\hline III & 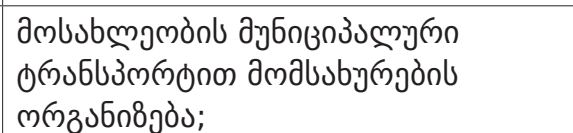 & 2,9 & III & 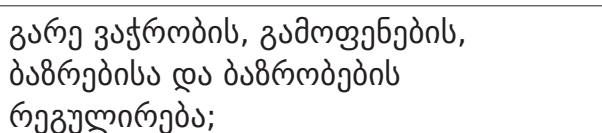 & 2,4 \\
\hline
\end{tabular}




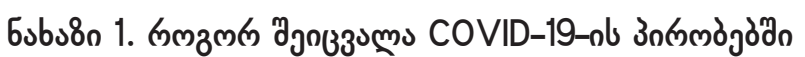
bufanobmò?

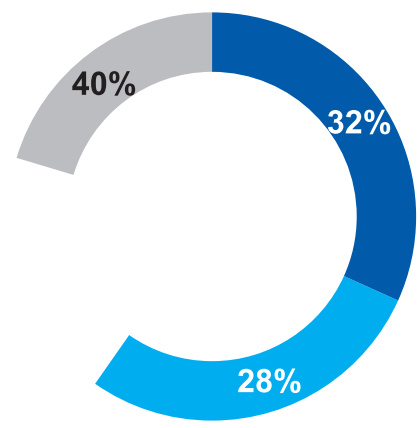

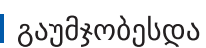

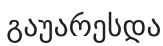

juscosmos

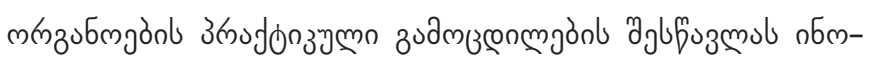

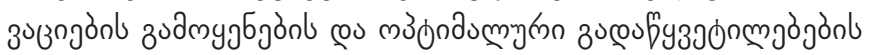
angुön क ऊumbus 8 monlon;

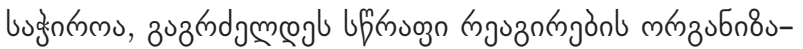

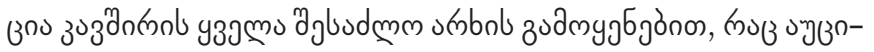

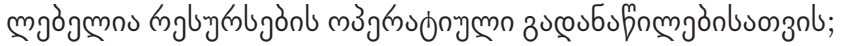

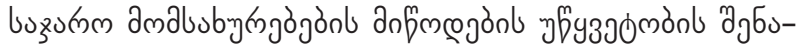

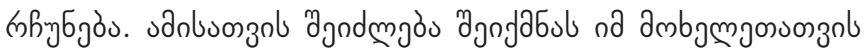

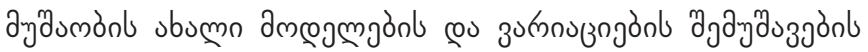

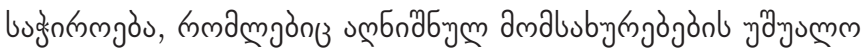

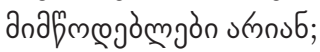

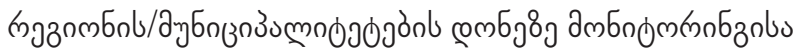

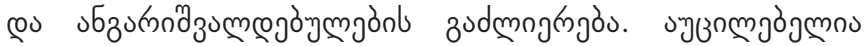

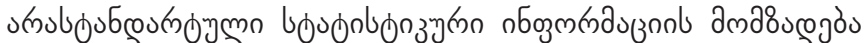

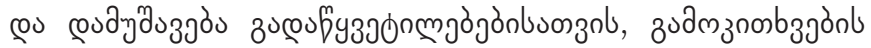

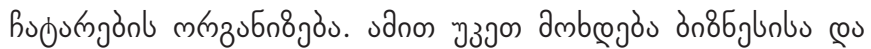

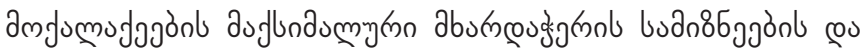

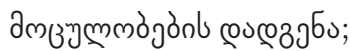

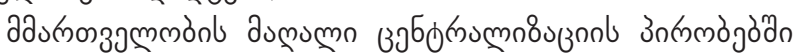

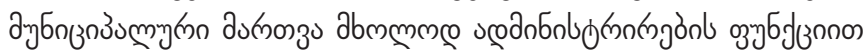

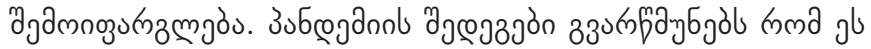

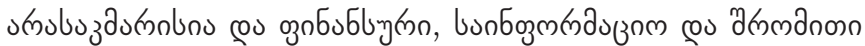

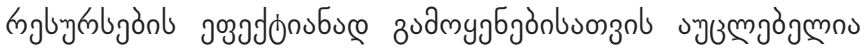

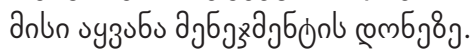

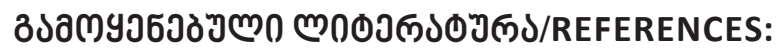

Böckenförde, M. (2011). A Practical Guide to Constitution Building: Decentralized Forms of Government, 4; 18.2011. URL:https://www.idea.int/sites/default/files/publications/chapters/practical-guide-to-constitution-building/apractical-guide-to-constitution-building-chapter-5.pdf

Chikhladze, N., Chikhladze N., \& Rusadze N. (2021). Challenges of Economic Activities of Cities in the Context of the Covid-19 Pandemic. Bulletin of the Georgian National Academy of Sciences, 15, (1) 110-116. http://science.org.ge/bnas/vol-15-1.html.

Civil Service Bureau Activity Report 2020. (in Georgian). URL:http://www.csb.gov.ge/media/3168/7447.pdf

COVID-19: Embracing digital government during the pandemic and beyond. United Nations Di-vision for Public Institutions and Digital Government. UN/DESA Policy Brief, 61. URL:https://www.un.org/development/desa/dpad/publication/undesa-policy-brief-61-covid-19-embracing-digital-government-during-the-pandemic-and-beyond/

LocalSelf-GovernmentCode,Article16.(inGeorgian).URL:https://matsne.gov.ge/ka/document/view/2244429?publication=57 Materials of the Economic Development, Local Self-Government Property and Transport Management Service of Kutaisi Municipality. (in Georgian).

National Accounts of Georgia (2019). National statistics office of Georgia. URL:https://www.geostat.ge/media/37666/\%E1\%8 3\%99\%E1\%83\%A0\%E1\%83\%94\%E1\%83\%91\%E1\%83\%A3\%E1\%83\%9A\%E1\%83\%98-2019.pdf

OECD (2019) Making Decentralization Work, a Handbook for Policy-Makers, pp-3;6. URL:https://www.oecd.org/regional/ making-decentralisation-work-g2g9faa7-en.htm

Ordinance 269 of Kutaisi Municipality Council, May 27, 2020; Ordinance 289 of the Kutaisi Municipality Council, September 30, 2020. (in Georgian).

Papava, V., \& Silagadze, A. (2019). On the Georgian Name of the Key Economic Term"Gross Domestic Product." Journal Economics and Business, 1, 180-182.

Shergelashvili T., (2017) Continuation of the territorial changes of the municipality, which started in 2014, is extremely important for the development of the country. (in Georgian). URL: URL:http://droa.ge/?p=4143

Sitizen's stratification with local public services in Georgia (2013). UNDP, SDS, ADC, 2013. 